Article

\title{
TRAIL Deficient Mice Are Protected from Sugen/Hypoxia Induced Pulmonary Arterial Hypertension
}

\author{
Sarah H. Dawson ${ }^{\dagger}$, Nadine D. Arnold ${ }^{\dagger}$, Josephine A. Pickworth, Sheila E. Francis and
}

Allan Lawrie *

Department of Cardiovascular Science, University of Sheffield, Medical School, Beech Hill Road, Sheffield, S10 2RX, UK; E-Mails: mda08sd@sheffield.ac.uk (S.H.D.);n.d.arnold@sheffield.ac.uk (N.D.A.); j.a.pickworth@sheffield.ac.uk (J.A.P.); s.francis@sheffield.ac.uk (S.E.F.)

$\dagger$ These authors contributed equally to this work.

* Author to whom correspondence should be addressed; E-Mail: a.lawrie@sheffield.ac.uk; Tel.: +44-114-271-1376; Fax: +44-114-271-1863.

Received: 11 March 2014; in revised form: 15 July 2014 / Accepted: 28 July 2014 /

Published: 31 July 2014

Abstract: Pulmonary arterial hypertension (PAH) is a progressive lung disease diagnosed by an increase in pulmonary arterial blood pressure that is driven by a progressive vascular remodelling of small pulmonary arterioles. We have previously reported that tumor necrosis factor apoptosis-inducing ligand (TRAIL) protein expression is increased in pulmonary vascular lesions and pulmonary artery smooth muscle cells (PASMC) of patients with idiopathic PAH. The addition of recombinant TRAIL induces the proliferation and migration of PASMCs in vitro. TRAIL is required for hypoxia-induced pulmonary hypertension in mice, and blockade of TRAIL prevents and reduces disease development in other rodent models of PAH. Due to the availability of knockout and transgenic mice, murine models of disease are key to further advances in understanding the complex and heterogeneous pathogenesis of PAH. However, murine models vary in their disease severity, and are often criticized for lacking the proliferative pulmonary vascular lesions characteristic of PAH. The murine Sugen-hypoxic (SuHx) mouse model has recently been reported to have a more severe PAH phenotype consisting advanced pulmonary vascular remodelling. We therefore aimed to determine whether TRAIL was also required for the development of PAH in this model. C57BL/6 and $\mathrm{TRAIL}^{-1-}$ mice were exposed to normoxia, Sugen5416 alone, hypoxia or both Sugen5416 and hypoxia (SuHx). We report here that SuHx treated C57BL/6 mice developed more severe PAH than 
hypoxia alone, and that $\mathrm{TRAIL}^{-/-}$mice were protected from disease development. These data further emphasise the importance of this pathway and support the use of the SuHx mouse model for investigating the importance of potential mediators in PAH pathogenesis.

Keywords: TRAIL; murine model; pulmonary arterial hypertension; sugen/hypoxia

\section{Introduction}

Pulmonary hypertension (PH) is a broad group of diseases defined by a mean pulmonary artery pressure (mPAP) of greater than or equal to $25 \mathrm{mmHg}$ at right heart catheterisation [1]. Pulmonary arterial hypertension (PAH) is a sub-classification of $\mathrm{PH}$ and a progressive and degenerative disease driven by progressive arterial remodelling within the pulmonary circulation [2]. PAH is a rare disease, with an incidence of 1-3.3 per million per year (idiopathic PAH) and a prevalence of 15-52 per million [1] but has severe morbidity, a prognosis worse than many cancers and is ultimately fatal (without lung transplantation). Although PAH is a disease of the lungs, it is the impact on the right ventricle which ultimately defines a patients survival. The increased pulmonary vascular resistance results in a pressure afterload that drives a process of adaptive, then maladaptive right ventricular hypertrophy, dilation and stiffening. This leads to impaired stroke volume, inadequate organ perfusion and eventual right heart failure [3]. Current treatments for PAH are effective in alleviating vasoconstrictive symptoms, however they do not address the underlying pulmonary vascular or right ventricular remodelling and have only modest impact on patient survival.

The increase in pulmonary pressure observed in PAH is largely due to underlying remodelling of small-to-medium sized pulmonary arterioles. The underlying mechanisms that lead to the pulmonary vascular remodelling are incredibly complex [4,5]. Although the exact trigger for pulmonary arteriole remodelling is currently unknown, pulmonary arterial endothelial cell (PA-EC) dysfunction and apoptosis is believed to be an early insult. PA-EC apoptosis causes the obliteration of distal pulmonary arterioles and exposes the underlying pulmonary arterial smooth muscle cells (PA-SMCs) to a whole host of mitogenic and vasoconstrictive factors, which then drive the vascular remodelling [4,5]. A population of dysfunctional, hyper-proliferative PA-ECs also proliferate to form plexiform lesions, a hallmark of severe PAH [6]. Growth factors, such as PDGF [7,8] and cytokines such as IL-1 and IL-6 [9-13] have also been implicated in the pathogenesis of PAH. More recently, we have described TRAIL as a critical downstream mediator of disease [14].

TRAIL (TNF-related apoptosis inducing ligand, Apo2L) is a type two membrane protein that is predominantly expressed in the lung, spleen and prostate [15]. TRAIL binds to four membrane receptors: TRAIL R1 (death receptor 4, DR4) [16], TRAIL R2 (death receptor 5, DR5) [16,17], TRAIL R3 (decoy receptor 1, DcR1) [16,18] and TRAIL R4 (decoy receptor 2, DR2) [19,20]. There is also a fifth, soluble decoy receptor, osteoprotegerin (OPG) [21], a putative biomarker for IPAH [22]. TRAIL induces the proliferation and migration of both rat and human aortic SMCs, and human pulmonary artery smooth muscle cells predominately via TRAIL-R3 [14,23]. TRAIL protein expression has also been observed in concentric and plexiform pulmonary vascular lesions in patients with IPAH [24] and in animal models of disease [11,14]. In rodent models anti-TRAIL antibody 
prevents disease development [14]. Furthermore, in therapeutic studies, where antibody treatment was performed in models with established disease, an anti-TRAIL antibody successfully reduced pulmonary vascular remodelling and increased survival [14].

Due to the availability of knockout and transgenic mice, murine models of disease are key to further advances in understanding the complex and heterogeneous pathogenesis of PAH. The most common murine model is the chronic hypoxic mouse model [25], however the mouse is a poor responder to hypoxia in comparison to other species, and therefore often criticized as a poor model of PAH [26]. More recently, an adaptation of the rat Sugen-hypoxic (SuHx) model, first developed by Taraseviciene-Stewart and colleagues [27], to mice has been shown to display a more severe disease phenotype, with higher right heart pressure and more severe pulmonary vascular remodelling than hypoxia alone [28]. We therefore aimed to both establish the murine model of SuHx and hypothesised that $\mathrm{TRAIL}^{-1}$ mice would be protected from developing PAH in this model. We found that while C57BL/6 mice developed the hallmarks of PAH including remodelling of the small pulmonary arterioles, increased right heart pressure and right ventricular hypertrophy, $\mathrm{TRAIL}^{-/-}$mice showed no significant signs of PAH.

\section{Experimental Section}

\subsection{Animals}

All mice were on a C57BL/6 background. TRAIL ${ }^{-/}$mice were originally developed by Amgen/Immunex (Thousand Oaks, CA, USA.) as previously described [29,30] and obtained by material transfer agreement from Amgen Inc. (MTA \# 200908042). For all procedures male C57BL/6 and $\mathrm{TRAIL}^{-/}$mice aged 12-13 weeks of age were used (6-8 per group). For the chronic hypoxic mouse model, mice were placed in hypoxic chambers $(10 \% \mathrm{v} / \mathrm{v}$ Oxygen) for 3 weeks to induce pulmonary hypertension. For the Sugen-hypoxic model, C57BL/6 and TRAIL ${ }^{-1-}$ were exposed to hypoxia $\left(10 \% \mathrm{v} / \mathrm{v} \mathrm{O}_{2}\right)$ for 3 weeks with weekly injections of $20 \mathrm{mg} / \mathrm{kg}$ Sugen5416 (Tocris) during exposure to hypoxia as previously described [28]. All animal experiments were approved by the University of Sheffield Project Review Committee and conformed to UK Home Office ethical guidelines.

\subsection{Echocardiography}

Echocardiography was performed using the Vevo 770 system (VisualSonics, Toronto, Canada) using the RMV707B scan head. Rodents were placed on a heated platform and covered to minimise heat loss. Rectal temperature, heart rate and respiratory rate were recorded continuously throughout the study. Echocardiography was performed and analysed as previously described $[11,14]$.

\subsection{Cardiac Catheterisation}

Following echocardiography, left and right ventricular catheterisation was performed using a closed chest method via the right internal carotid artery and right external jugular vein under isoflurane induced anaesthesia, as previously described [11,14]. Data were collected using either a Millar pressure-volume PVR-1045 1F catheter (mouse LV) or PVR-1030 (mouse RV) (Millar Instruments Inc., Texas, USA) coupled to a Millar MPVS 300 and a PowerLab 8/30 data acquisition system (AD 
Instruments, Oxfordshire, UK) and recorded using Chart v7 software (AD Instruments). Pressure volume analysis was performed (PVAN, Millar) and an estimated pulmonary vascular resistance index (ePVRi) was calculated as previously described [14].

\subsection{Right Ventricular Hypertrophy}

Right ventricular hypertrophy (RVH) was measured by calculating the ratio of the right ventricular free wall over left ventricle plus septum [14].

\subsection{Immunohistochemistry}

Immediately after harvest, the left lung was perfusion fixed via the trachea with $10 \%(\mathrm{v} / \mathrm{v})$ formal buffered saline by inflation to $20 \mathrm{~cm}$ of $\mathrm{H}_{2} \mathrm{O}$. The lungs were then processed into paraffin blocks for sectioning. Paraffin embedded sections $(5 \mu \mathrm{m})$ of lung were histologically stained for Alcian Blue Elastin van Gieson (ABEVG) and immunohistochemically stained for $\alpha$-smooth muscle actin ( $\alpha$-SMA, M0851, Dako, Cambridgeshire, UK) to visualize smooth muscle cells, TRAIL (TRAIL (ab2435; Abcam) was also used to localize protein expression to pulmonary vascular lesions and anti-human PCNA antibody to assess proliferation (M0879; Dako). A secondary biotinylated anti-mouse antibody (1:200) was added before an Avidin Biotin enzyme Complex (Vectastain kit;Vector Laboratories). All protocols used 3,3 diaminobenzidine as the substrate for color in the peroxidase reaction and counterstained with hematoxylin as previously described [14].

\subsection{Quantification of Pulmonary Vascular Remodelling}

Pulmonary vascular remodelling was quantified by assessing the degree of muscularization and the percentage of affected pulmonary arteries and arterioles in 3 groups based on vessel size; small pulmonary arterioles with a diameter less than $50 \mu \mathrm{m}$, medium pulmonary arteries with a range in diameter from 51 to $100 \mu \mathrm{m}$, and large pulmonary arteries with a diameter greater than $100 \mu \mathrm{m}$ and standard immunohistochemical techniques were applied as previously described $[11,14]$.

\subsection{Western Immunoblotting}

$30 \mu \mathrm{g}$ of each sample was loaded on a $4 \%-12 \%$ Bis-Tris NuPage gel and run under reducing conditions in MES running buffer (Invitrogen), before transfer to a nitrocellulose membrane (Invitrogen). Trans- fer was confirmed with Ponceau S (Sigma-Aldrich) staining and the membrane was then blocked for $1 \mathrm{~h}$ in Odyssey ${ }^{\circledR}$ blocking buffer (LI-COR Biosciences) at room temperature. The blots were incubated with either GAPDH $(1: 1,000, \mathrm{CST})$ or anti-beta-Actin mouse monoclonal antibody $(1: 1,000$, Abcam) for $1 \mathrm{~h}$ at room temperature and anti-TRAIL mouse monoclonal antibody (1:50, Leica Biosystems) or anti-BMPR2 mouse monoclonal antibody $(1: 250)$, overnight at $4{ }^{\circ} \mathrm{C}$. For detection, membranes were incubated with fluorescently labeled anti-rabbit (IRDye $800 \mathrm{CW}$; LI-COR Biosciences) and anti-mouse (IRDye 680LT) for $1 \mathrm{~h}$ at room temperature before reading at 700/800 $\mathrm{nm}$ on an Odyssey SA imaging system (LI-COR Biosciences). 


\subsection{Statistical Analysis}

Statistical analysis was performed using two-way ANOVA followed by Dunnett's or Tukey's multiple comparisons test with a $95 \%$ confidence level. $\mathrm{P}<0.05$ was deemed statistically significant.

\section{Results and Discussion}

\subsection{BMPR2 Expression Is Decreased and TRAIL Expression Is Increased in the SuHx Model of PAH}

To determine whether we could recapitulate molecular characteristics of the SuHx model and whether this model was suitable to investigate the role of TRAIL in PAH vascular remodelling, we first looked to determine whether BMPR2 expression was decreased, and TRAIL expression increased within the lungs. After 3 weeks exposure to Sugen5416 plus hypoxia BMPR2 protein levels were significantly reduced compared to normoxic control C57Bl/6 (wt) mice (Figure 1A). On the contrary, TRAIL protein expression was significantly increased in whole lungs lysates from hypoxia and SuHx-treated wt mice, compared with normoxic or Sugen-alone treated mice (Figure 1B). TRAIL protein expression was also detected within remodelled pulmonary arteries of hypoxic and SuHx wt mice (Figure 1C).

Figure 1. BMPR2 expression is reduced and TRAIL expression is increased in the $\mathrm{SuHx}$ mouse model. Bar graph shows (A) reduced BMPR2 expression and (B) increased TRAIL protein expression in whole lung protein lysates of $\mathrm{C} 57 \mathrm{Bl} / 6$ mice following 3-week exposure to normoxia $(\mathrm{Nx})$, Sugen-alone $(\mathrm{Su})$, hypoxia-alone $(\mathrm{Hx})$ and Sugen plus Hypoxia (SuHx). Representative western immunoblot images are also shown. (C) Representative photomicrographs of lung sections of SuHx mice showing immunoreactivity for TRAIL. Arrows highlight TRAIL positive cells within remodelled vessels. Error bars represent mean $\pm \mathrm{SEM}, * \mathrm{P}<0.05$, ** $\mathrm{p}<0.01 ; \mathrm{n}=3-4$ animals per group. Scale bars represent $50 \mu \mathrm{m}$.

A
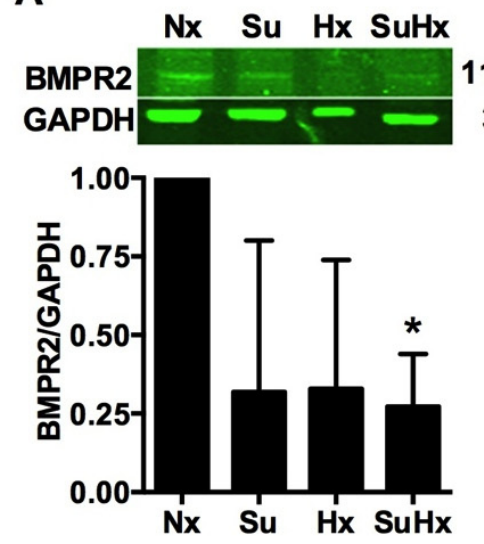

B

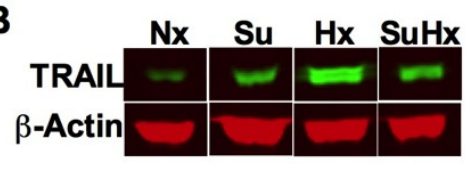

$32 \mathrm{kDa}$

$42 \mathrm{kDa}$

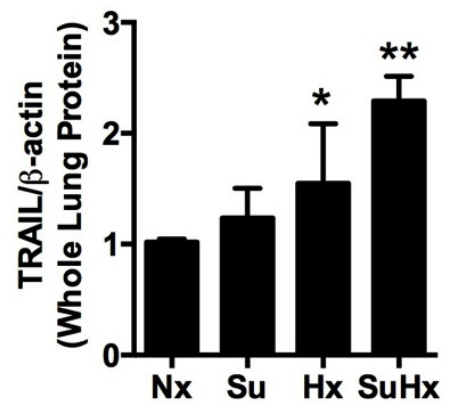

C

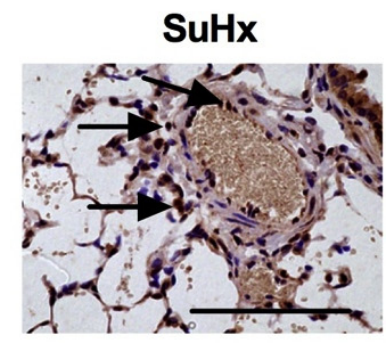

\subsection{TRAIL ${ }^{-/}$Mice Show no Echocardiographic Signs of PAH in Response to SuHx}

Compared to control normoxic, Sugen-alone and hypoxia-alone treated mice, wt mice exposed to SuHx displayed outward signs of morbidity including breathlessness, consistent with a PAH 
phenotype. Serial echocardiography performed on wt mice exposed to SuHx displayed a PAH phenotype, as defined by a reduction in pulmonary artery acceleration time (PA-AT) (Figure 2A), cardiac output (Figure 2B), pulmonary artery velocity time integral (PA-VTi) (Figure 2E) and an increased right ventricular internal diameter at diastole (RVIDd) (Figure 2D). Hypoxia- and SuHx-treated $\mathrm{TRAIL}^{-/-}$mice however, displayed no significant change in either parameter compared to baseline or normoxic control mice. Both wt and $\mathrm{TRAIL}^{-/-}$hypoxic mice did however show a significant decrease in aortic-VTi (Figure 2F), presumably due to hypoxia mediated peripheral vasodilation [31].There were however no significant changes observed to the left ventricular wall, LV FWTs (Figure 2C) and LV FWTd (Figure 2G). The SuHx-treated wt mice were the only group to display a significant increase in RVIDd (Figure 2D). There was significant increase in RVH from both hypoxia and SuHx treated wt mice that was not observed in the TRAIL ${ }^{--}$mice. Although there was a trend for increased RVH between SuHx and hypoxia alone wt mice, this was not statistically significant (Figure 2H).

Figure 2. TRAIL ${ }^{-/}$mice show no echocardiographic signs of PAH in response to SuHx. (A-H) Bar graphs show (A) pulmonary artery acceleration time (PA-AT), (B) cardiac output, (C) left ventricle free wall thickness at systole (LV FWTs), (D) right ventricular internal diameter at diastole (RVIDd), (E) pulmonary artery velocity time integral (PA-VTi), (F) aortic velocity time integral Ao-VTi), (G) left ventricle free wall thickness at diastole (LV FWTd) and (H) right ventricular hypertrophy $(\mathrm{RVH})$ in wt and TRAIL ${ }^{-/-}$mice at baseline and after 3 weeks exposure to normoxia, Sugen5416, hypoxia or SuHx. Error bars represent mean \pm SEM, $\mathrm{n}=4-6$ animals in each group. $* \mathrm{P}<0.05$; ** $\mathrm{P}<0.01 ; * * * \mathrm{P}<0.001$, wt mice compared to wt baseline. ${ }^{*} \mathrm{P}<0.05$, TRAIL $^{-/-}$compared to TRAIL ${ }^{-/-}$baseline.

A

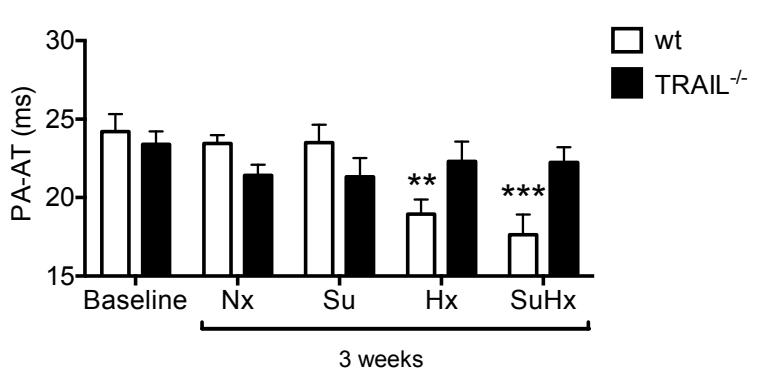

B

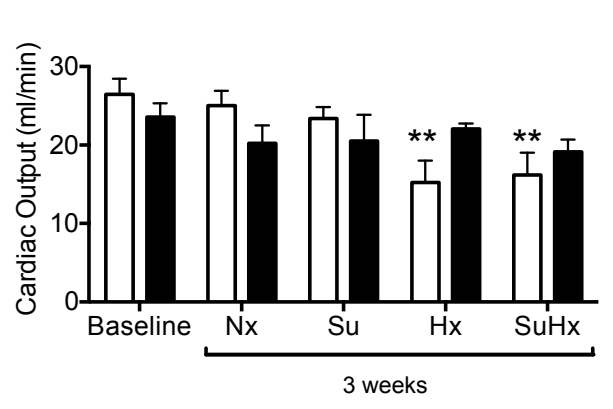

E

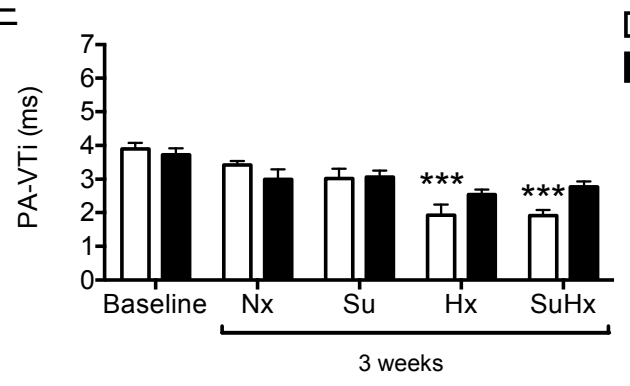

F

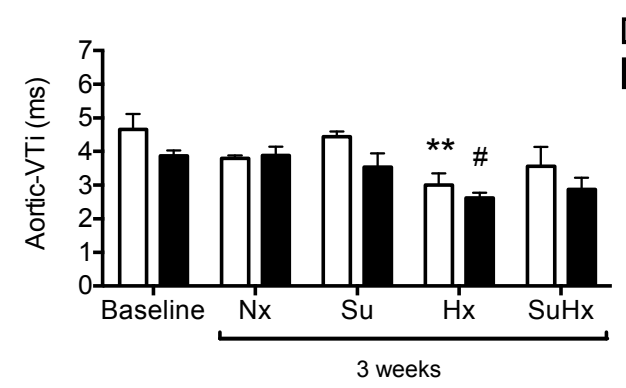


Figure 2. Cont.
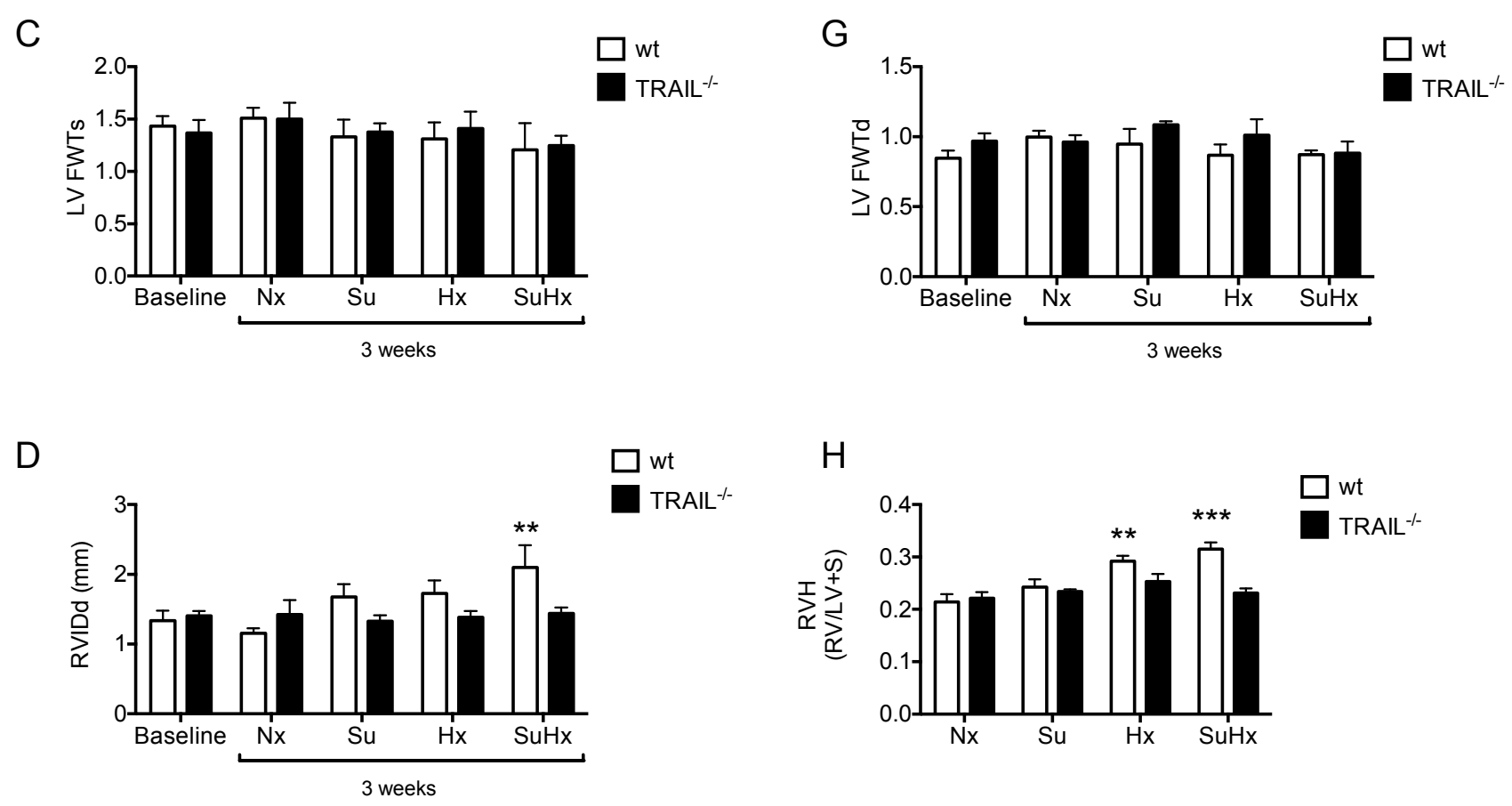

\subsection{TRAIL ${ }^{-/-}$Mice Show no Significant Haemodynamic Signs of PAH in Response to SuHx}

Following echocardiography assessment, right and left ventricular catheterisation was performed. Wild-type mice exposed to hypoxia and SuHx developed significant increases in RVSP (Figure 3A), $\mathrm{RV} \mathrm{dP} / \mathrm{dt}_{\max }$ (Figure 3C), RV P@dP/dt $\mathrm{d}_{\max }$ (Figure 3E) and ePVRI (Figure 3M), and reduced cardiac output (Figure 3F), RV dV/dt (Figure 3D) and LV dV/dt (Figure 3K). In contrast to wt mice, hypoxiaand SuHx-treated $\mathrm{TRAIL}^{-/-}$mice did not develop significant changes in the haemodynamic parameters associated with disease development (Figure 3A-N). Wild-type SuHx-treated mice had significantly increased RVSP (Figure 3A) and ePVRi (Figure 3M) compared to hypoxia alone, and were the only group to display a significant increase in right ventricular pressure at max $\mathrm{dP} / \mathrm{dt}$ (Figure $3 \mathrm{E}$ ) suggesting a more severe PAH phenotype similar to that reported by Ciuclan et al. [28].

\subsection{TRAIL ${ }^{-/-}$Mice Show Reduced Pulmonary Vascular Remodelling in Response to SuHx}

To examine whether the more severe PAH phenotype in SuHx wt mice, compared to hypoxia alone, was due to more extensive pulmonary vascular remodelling, and whether the $\mathrm{TRAIL}^{-/}$mice were protected from pulmonary vascular changes, we performed quantitative analysis of serial lung sections. To assess the degree of pulmonary vascular remodelling, we measured the media cross sectional area (media/CSA) and degree of muscularization in pulmonary arteries of $<50 \mu \mathrm{m}, 50-100 \mu \mathrm{m}$ and $>100 \mu \mathrm{m}$ in diameter as previously described [11,14]. Wild-type mice showed increased media/CSA (Figure 4A) and a greater percentage of muscularized arteries (Figure 4B) in pulmonary arterioles of $<50 \mu \mathrm{m}$ diameter when treated with hypoxia alone or SuHx, compared to normoxic controls. Although there was no significant difference between hypoxia and SuHx wt mice with either index, there were trends for both a higher percentage of remodelled vessels, and increased medial area. The more severe haemodynamics observed in the wt SuHx was likely due to the more progressive nature of the 
pulmonary vascular remodelling as demonstrated by the significant increase in media/CSA (Figure 4D), and percentage remodelling (Figure 4E) of the medium sized 50-100 $\mu \mathrm{m}$ pulmonary arteries, which was not observed in the hypoxia alone wt mice. No difference in media/CSA, or in percentage muscularization was observed in pulmonary arterioles of $>100 \mu \mathrm{m}$ (Figure $4 \mathrm{G}, \mathrm{H}$ ). TRAIL ${ }^{-/-}$mice displayed no significant increases in either marker of pulmonary vascular remodelling in response to either hypoxia or SuHx (Figure 4A-F). To verify that the increased vascular remodelling was driven by pulmonary vascular cell proliferation, we performed immunohistochemical analysis of proliferating cell nuclear antigen (PCNA). There was little difference between wt and TRAIL ${ }^{-/}$mice in response to hypoxia, perhaps highlighting that the major driver of apparent muscularisation of the pulmonary arteries was more vasoconstriction, rather than aberrant proliferation (Figure 4C,F,I). Within the SuHx wt mice however, there was a marked and significant increase in the number of proliferating cells within the remodelled vessels (Figure 4 C,F,I).

Figure 3. TRAIL ${ }^{-/}$mice show no haemodynamic signs of PAH in response to SuHx. (A-N) Bar graphs show (A) right ventricular systolic pressure (RVSP), (B) right ventricular end-diastolic pressure (RVEDP), (C) right ventricular $(\mathrm{RV}) \mathrm{dP} / \mathrm{dt}$, (D) right ventricular $(\mathrm{RV}) \mathrm{dV} / \mathrm{dt}$, (E) right ventricular (RV) P@dP/dt, (F) cardiac output, (G) mean aortic blood pressure $(\mathrm{mAoP}),(\mathbf{H})$ left ventricular end-systolic pressure (LVESP), (I) left ventricular end-diastolic pressure (LVEDP), (J) left ventricular (LV) dP/dt, (K) left ventricular (LV) $\mathrm{dV} / \mathrm{dt}$, (L) left ventricular (LV) P@dP/dt, (M) estimated pulmonary vascular resistance (ePVRi) and (N) systemic vascular resistance (SVR) in wt and TRAIL ${ }^{-/}$mice after 3 weeks exposure to normoxia, Sugen5416, hypoxia or SuHx. Error bars represent mean \pm SEM, $\mathrm{n}=3-6$ animals in each group. * $\mathrm{P}<0.05 ; * * \mathrm{P}<0.01$; **** $\mathrm{P}<0.0001$, compared to wt normoxic mice, significant differences between other groups are shown.
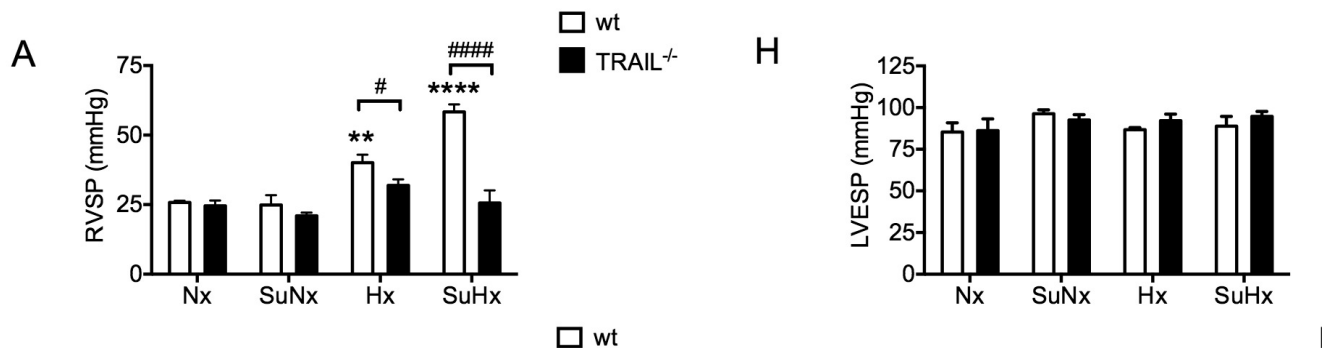

B
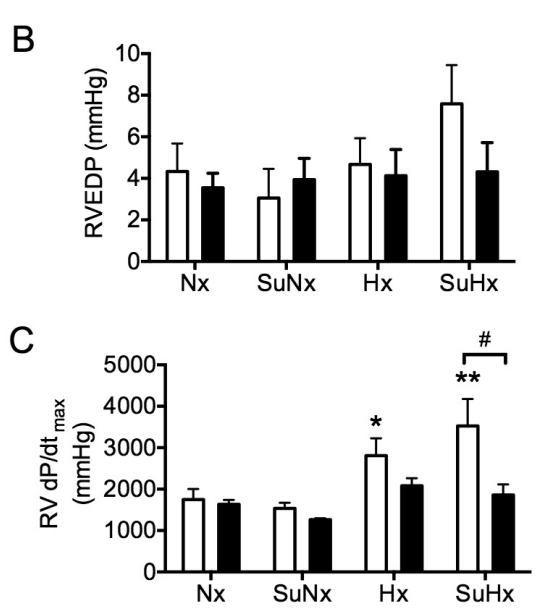

$\square$ wt TRALL $^{-1} \quad$ I

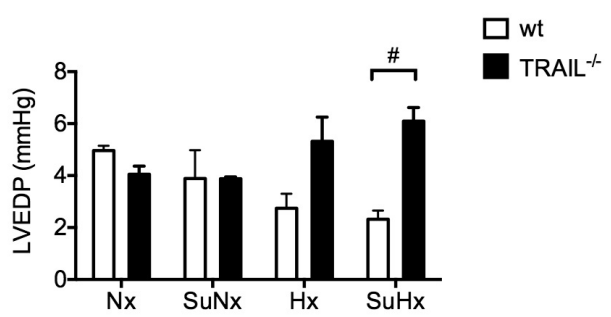

$\square$ wt $\quad$ JRAL ${ }^{-1-} \quad J$

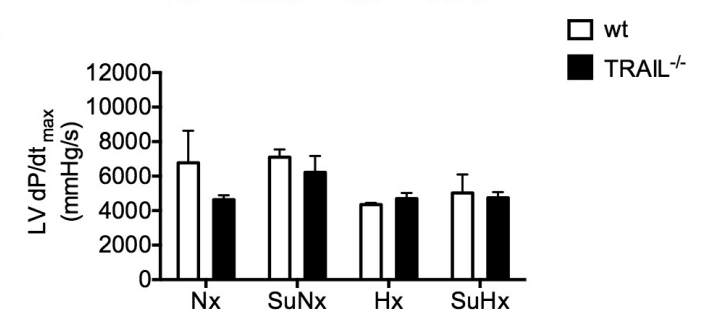


Figure 3. Cont.

D

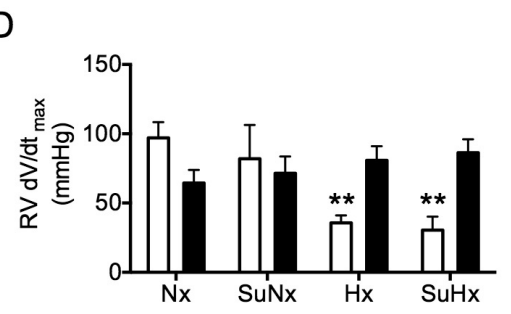

E

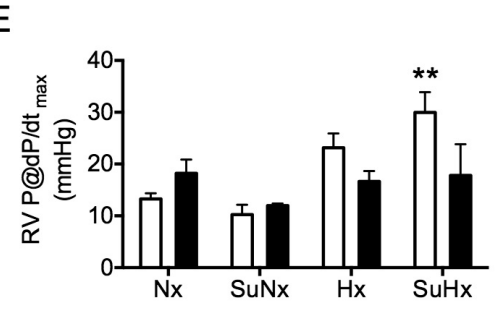

F

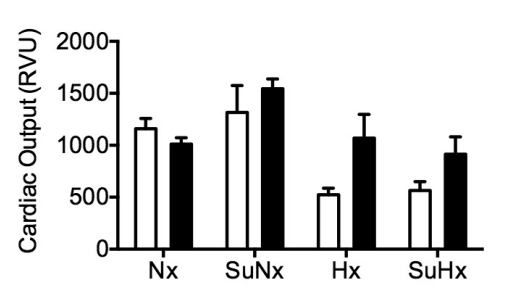

G

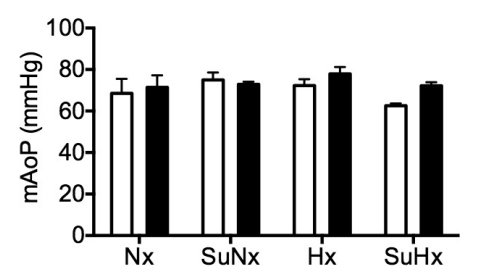

K

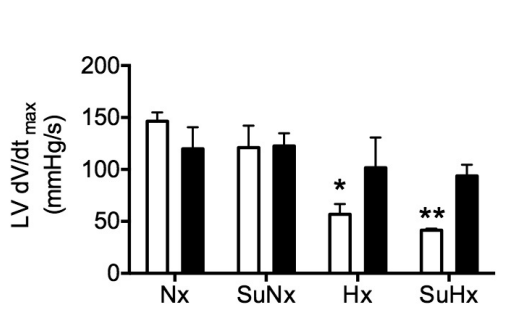

L

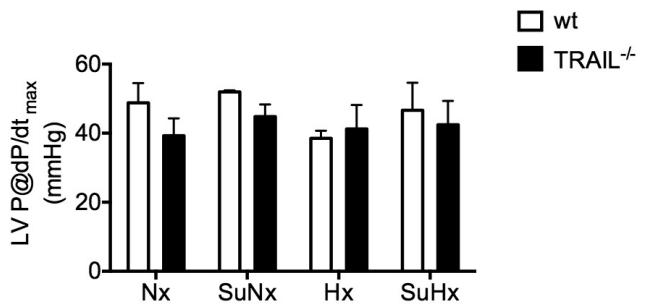

M

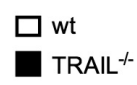

$M$

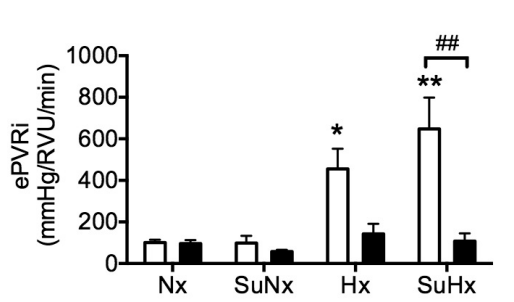

$\mathrm{N}$

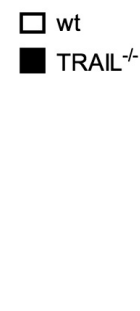

$\square \mathrm{wt}$

[ TRAIL $^{-1}$

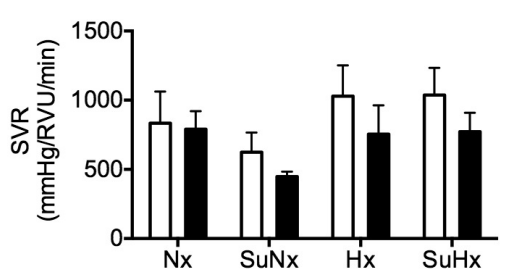

$\square \mathrm{wt}$

TRAIL - -

$\square$ wt

- TRAIL $^{-1}$

Histological and immunohistological analysis of lung tissues (Figure 5) revealed evidence of muscularization caused by increased smooth muscle actin (SMA) positive cells in small resistance pulmonary arterioles, $<50 \mu \mathrm{m}$ diameter, in hypoxic and $\mathrm{SuHx}$ mice compared to normoxic mice. Although more extensive pulmonary vascular remodelling was observed in the SuHx treated wt mice we did not observe any evidence of plexiform-like lesions. TRAIL ${ }^{-1-}$ mice displayed reduced remodelling of the pulmonary arterioles in response to both hypoxia and SuHx that corresponded with the presence or absence of proliferating cells (PCNA, Figure 5).

\subsection{The Protection from Pulmonary Vascular Remodelling in TRAIL ${ }^{-/-}$Mice in the SuHx Model Is Associated with Reduced Recruitment of Inflammatory Cells}

Previous studies in chimeric mice suggest that tissue expression of TRAIL is the predominant driver of pulmonary remodelling in the high fat diet fed $\mathrm{ApoE}^{-/-}$mouse model [14], however the contribution of bone marrow (BM)-derived cells could not be entirely ruled out. To determine whether protection from the development of a PAH phenotype was also associated with a reduced inflammatory cell infiltration, we performed immunohistochemical analysis for CD45 positive cells in SuHx wt and TRAIL ${ }^{-/}$mice. While wt mice demonstrated a marked increase in CD45 positive cells in response to SuHx, $\mathrm{TRAIL}^{-1-}$ mice had a similar expression profile to control wt mice (Figure 6). 
Figure 4. TRAIL ${ }^{-/-}$mice show reduced pulmonary vascular muscularization in response to SuHx. Bar graphs show media/CSA of pulmonary arteries (A) $<50 \mu \mathrm{m}$ diameter, (D) 50-100 $\mu \mathrm{m}$ diameter, $(\mathbf{G})>100 \mu \mathrm{m}$ diameter; the percentage of muscularized arteries (B) $<50 \mu \mathrm{m}$ diameter, (E) 50-100 $\mu \mathrm{m}$ diameter, (H) $>100 \mu \mathrm{m}$ diameter; percentage of proliferating cells within pulmonary arteries (C) $<50 \mu \mathrm{m}$ diameter, (F) $50-100 \mu \mathrm{m}$ diameter, (I) $>100 \mu \mathrm{m}$ diameter in wt and $\mathrm{TRAIL}^{-/-}$mice exposed to normoxia $(\mathrm{Nx})$, sugen-alone $(\mathrm{Su})$, hypoxia $(\mathrm{Hx})$ and Sugen plus Hypoxia $(\mathrm{SuHx})$. Error bars represent mean \pm SEM, $\mathrm{n}=3-6$ animals in each group. * $\mathrm{P}<0.05$; ** $\mathrm{P}<0.01$; *** $\mathrm{P}<0.001$, compared to wt normoxic mice.

A

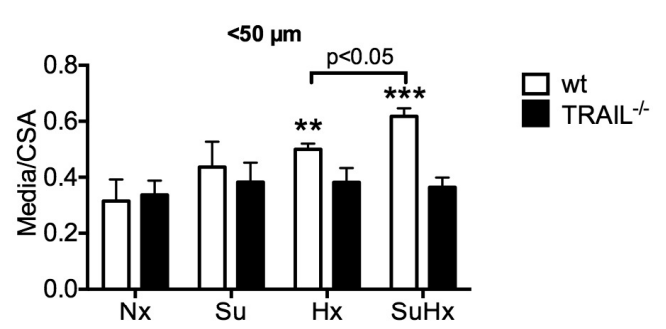

C

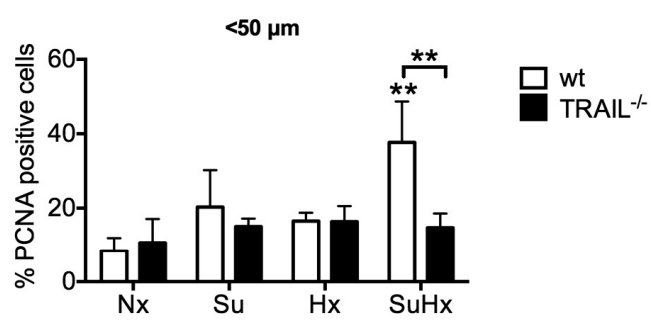

E

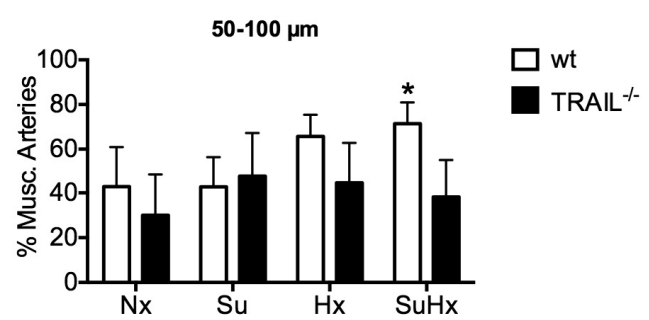

G
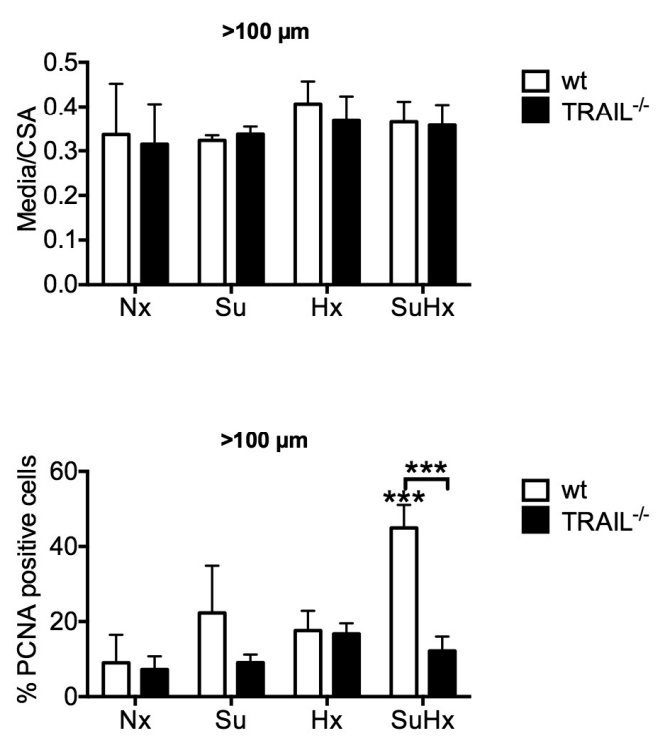

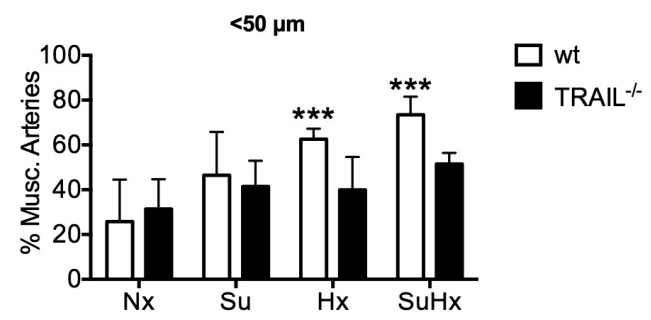

D

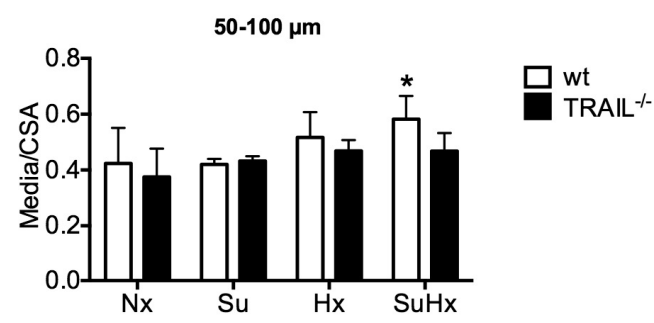

F

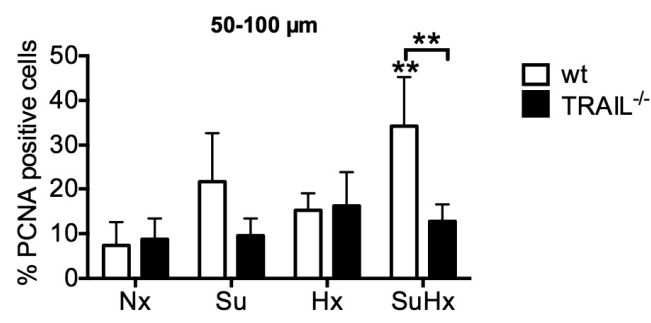

$\mathrm{H}$

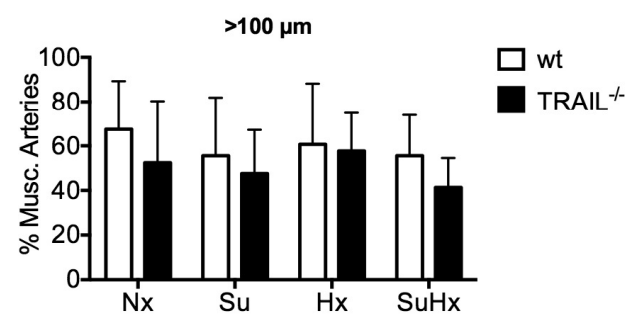


Figure 5. $\mathrm{TRAIL}^{-/}$mice are protected against small resistance pulmonary arterial muscularization when exposed to SuHx. Representative photomicrographs of lung sections from wt and $\mathrm{TRAIL}^{-/-}$mice after 3-week exposure to normoxia, Sugen 5416, hypoxia or SuHx. Sections were stained with Alcian Blue Elastic van Gieson (ABEVG), immunostained for $\alpha$-smooth muscle actin (SMA) or proliferating smooth muscle cell antigen (PCNA). Bars, $50 \mu \mathrm{m}$.
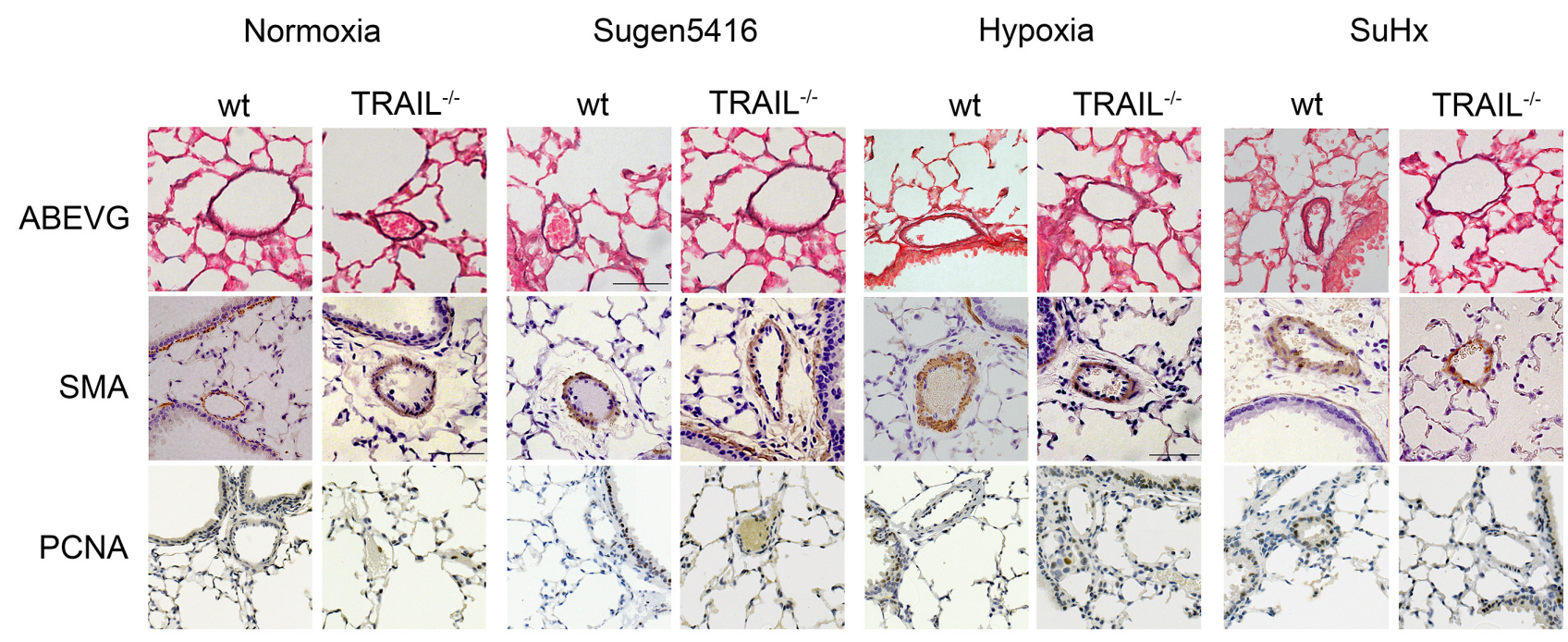

Figure 6. $\mathrm{TRAIL}^{-/}$recruit less CD45 positive cells when exposed to SuHx. Representative photomicrographs of lung sections from wt and $\mathrm{TRAIL}^{-/}$mice after 3-week exposure to Sugen plus hypoxia (SuHx). Sections were immunostained for CD45.

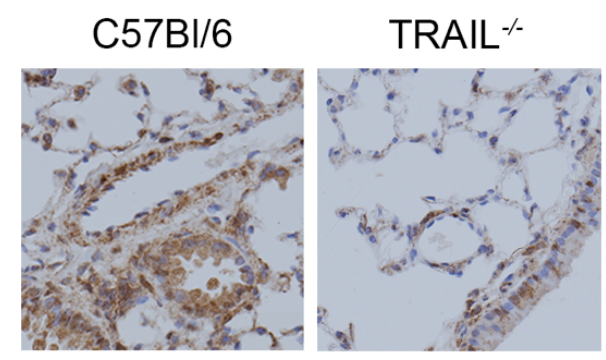

\section{Conclusions}

Rodent models, and particularly murine models of PAH, are often criticised for their limited ability to recapitulate or model human disease. Despite these caveats, the increasing availability of knockout and transgenic mice increases the importance of mouse models. The hypoxic mouse model has historically been the most common murine model [25] utilised despite the mouse being a poor responder to hypoxia, in comparison to other species, and therefore a weak model [26]. The recent adaptation of the rat Sugen-hypoxic (SuHx) [27] to mice by Ciuclan and colleagues [28] provides a more robust murine model in which to test the requirement, or importance of specific genes in disease pathogenesis.

We have previously demonstrated that $\mathrm{TRAIL}^{-/-}$mice are protected from hypoxia-induced $\mathrm{PH}$, and that $\mathrm{ApoE}^{-/-} / \mathrm{TRAIL}^{-/-}$are protected from high fat diet-induced PAH [14]. We therefore looked to test the utility of the murine SuHx model while simultaneously validating our own studies. We found that while C57BL/6 mice developed hallmarks of PAH as measured by echocardiography, cardiac 
catheterisation and remodelling of the small pulmonary arterioles as previously reported [28], TRAIL $^{-/-}$mice showed no significant signs of PAH, as evidenced by the reduced recruitment of inflammatory cells and reduced number of proliferating cells with the pulmonary arteries. These data emphasise the utility of the murine SuHx models as a tool to screen the importance of particular genes of interest, and further support the importance of TRAIL in the pathogenesis of PAH.

\section{Acknowledgments}

Funding for this study was provided by the Medical Research Council via a Doctoral Training Grant (S.D) and a Career Development Award (G0800318, A.L.). A.L. is currently supported by a British Heart Foundation Senior Basic Science Research Fellowship (FS/13/48/30453).

\section{Author Contributions}

S.D. collected and analysed data, and contributed to the writing and editing of the manuscript. N.A. collected and analysed data and helped edit the manuscript. J. P. collected and analysed data and helped edit the manuscript. S.F. obtained the $\mathrm{TRAIL}^{-/-}$mice from Amgen, and contributed to manuscript editing. A.L. Over looked all aspects of the project, collected and analysed data and contributed to manuscript writing.

\section{Conflicts of Interest}

The authors declare no conflict of interest.

\section{References}

1. Kiely, D.G.; Elliot, C.A.; Sabroe, I.; Condliffe, R. Pulmonary hypertension: Diagnosis and management. BMJ 2013, 346, f2028.

2. Tuder, R.M.; Abman, S.H.; Braun, T.; Capron, F.; Stevens, T.; Thistlethwaite, P.A.; Haworth, S.G. Development and pathology of pulmonary hypertension. J. Am. Coll. Cardiol. 2009, 54, S3-S9.

3. Vonk-Noordegraaf, A.; Haddad, F.; Chin, K.M.; Forfia, P.R.; Kawut, S.M.; Lumens, J.; Naeije, R.; Newman, J.; Oudiz, R.J.; Provencher, S.; et al. Right Heart Adaptation to Pulmonary Arterial Hypertension. J. Am. Coll. Cardiol. 2013, 62, D22-D33.

4. Rabinovitch, M. Molecular pathogenesis of pulmonary arterial hypertension. J. Clin. Invest. 2008, 118, 2372-2379.

5. Schermuly, R.T.; Ghofrani, H.A.; Wilkins, M.R.; Grimminger, F. Mechanisms of disease: pulmonary arterial hypertension. Nat. Rev. 2011, 8, 443-455.

6. Tuder, R.M.; Groves, B.; Badesch, D.B.; Voelkel, N.F. Exuberant endothelial cell growth and elements of inflammation are present in plexiform lesions of pulmonary hypertension. Am. J. Pathol. 1994, 144, 275-285.

7. Perros, F.; Montani, D.; Dorfmuller, P.; Durand-Gasselin, I.; Tcherakian, C.; Le Pavec, J.; Mazmanian, M.; Fadel, E.; Mussot, S.; Mercier, O.; et al. Platelet-derived growth factor expression and function in idiopathic pulmonary arterial hypertension. Am. J. Resp. Crit. Care Med. 2008, $178,81-88$. 
8. Schermuly, R.T.; Dony, E.; Ghofrani, H.A.; Pullamsetti, S.; Savai, R.; Roth, M.; Sydykov, A.; Lai, Y.J.; Weissmann, N.; Seeger, W.; et al. Reversal of experimental pulmonary hypertension by PDGF inhibition. J. Clin. Invest. 2005, 115, 2811-2821.

9. Humbert, M.; Monti, G.; Brenot, F.; Sitbon, O.; Portier, A.; Grangeot-Keros, L.; Duroux, P.; Galanaud, P.; Simonneau, G.; Emilie, D. Increased interleukin-1 and interleukin-6 serum concentrations in severe primary pulmonary hypertension. Am. J. Resp. Crit. Care Med. 1995, $151,1628-1631$.

10. Voelkel, N.; Tuder, R.; Bridges, J.; Arend, W. Interleukin-1 receptor antagonist treatment reduces pulmonary hypertension generated in rats by monocrotaline. Am. J. Resp. Cell Mol. Biol. 1994, 11, 664-675.

11. Lawrie, A.; Hameed, A.G.; Chamberlain, J.; Arnold, N.; Kennerley, A.; Hopkinson, K.; Pickworth, J.; Kiely, D.G.; Crossman, D.C.; Francis, S.E. Paigen diet-fed apolipoprotein E knockout mice develop severe pulmonary hypertension in an interleukin-1-dependent manner. Am. J. Pathol. 2011, 179, 1693-1705.

12. Soon, E.; Holmes, A.M.; Treacy, C.M.; Doughty, N.J.; Southgate, L.; Machado, R.D.; Trembath, R.C.; Jennings, S.; Barker, L.; Nicklin, P.; et al. Elevated levels of inflammatory cytokines predict survival in idiopathic and familial pulmonary arterial hypertension. Circulation 2010, 122, 920-927.

13. Steiner, M.K.; Syrkina, O.L.; Kolliputi, N.; Mark, E.J.; Hales, C.A.; Waxman, A.B. Interleukin-6 overexpression induces pulmonary hypertension. Circ. Res. 2009, 104, 236-244, 228.

14. Hameed, A.G.; Arnold, N.D.; Chamberlain, J.; Pickworth, J.A.; Paiva, C.; Dawson, S.; Cross, S.; Long, L.; Zhao, L.; Morrell, N.W.; et al. Inhibition of tumor necrosis factor-related apoptosisinducing ligand (TRAIL) reverses experimental pulmonary hypertension. J. Exp. Med. 2012, 209, 1919-1935.

15. Wiley, S.R.; Schooley, K.; Smolak, P.J.; Din, W.S.; Huang, C.P.; Nicholl, J.K.; Sutherland, G.R.; Smith, T.D.; Rauch, C.; Smith, C.A. Identification and characterization of a new member of the TNF family that induces apoptosis. Immunity 1995, 3, 673-682.

16. Pan, G. An Antagonist Decoy Receptor and a Death Domain-Containing Receptor for TRAIL. Science 1997, 277, 815-818.

17. Walczak, H.; DegliEsposti, M.; Johnson, R.; Smolak, P.; Waugh, J.; Boiani, N.; Timour, M.; Gerhart, M.; Schooley, K.; Smith, C.; et al. TRAIL-R2: A novel apoptosis-mediating receptor for TRAIL. EMBO J. 1997, 16, 5386-5397.

18. Degli-Esposti, M.; Smolak, P.; Walczak, H.; Waugh, J.; Huang, C.; DuBose, R.; Goodwin, R.; Smith, C. Cloning and characterization of TRAIL-R3, a novel member of the emerging TRAIL receptor family. J. Exp. Med. 1997, 186, 1165-1170.

19. Degli-Esposti, M.A.; Dougall, W.C.; Smolak, P.J.; Waugh, J.Y.; Smith, C.A.; Goodwin, R.G. The novel receptor TRAIL-R4 induces NF-kappaB and protects against TRAIL-mediated apoptosis, yet retains an incomplete death domain. Immunity 1997, 7, 813-820.

20. Pan, G.; Ni, J.; Yu, G.; Wei, Y.; Dixit, V. TRUNDD, a new member of the TRAIL receptor family that antagonizes TRAIL signalling. FEBS Lett. 1998, 424, 41-45. 
21. Emery, J.; McDonnell, P.; Burke, M.; Deen, K.; Lyn, S.; Silverman, C.; Dul, E.; Appelbaum, E.; Eichman, C.; DiPrinzio, R.; et al. Osteoprotegerin is a receptor for the cytotoxic ligand TRAIL. J. Biol. Chem. 1998, 273, 14363-14367.

22. Condliffe, R.; Pickworth, J.A.; Hopkinson, K.; Walker, S.J.; Hameed, A.G.; Suntharaligam, J.; Soon, E.; Treacy, C.; Pepke-Zaba, J.; Francis, S.E.; et al. Serum osteoprotegerin is increased and predicts survival in idiopathic pulmonary arterial hypertension. Pulmonary Circulation 2012, 2, $21-27$.

23. Kavurma, M.M.; Schoppet, M.; Bobryshev, Y.V.; Khachigian, L.M.; Bennett, M.R. TRAIL stimulates proliferation of vascular smooth muscle cells via activation of NF-kappaB and induction of insulin-like growth factor-1 receptor. J. Biol. Chem. 2008, 283, 7754-7762.

24. Lawrie, A.; Waterman, E.; Southwood, M.; Evans, D.; Suntharalingam, J.; Francis, S.; Crossman, D.; Croucher, P.; Morrell, N.; Newman, C. Evidence of a role for osteoprotegerin in the pathogenesis of pulmonary arterial hypertension. Am. J. Pathol. 2008, 172, 256-264.

25. Lawrie, A. A report on the use of animal models and phenotyping methods in pulmonary hypertension research. Pulmonary Circulation 2014, doi:10.1086/674886.

26. Stenmark, K.R.; Meyrick, B.; Galie, N.; Mooi, W.J.; McMurtry, I.F. Animal models of pulmonary arterial hypertension: the hope for etiological discovery and pharmacological cure. Am. J. Physiol. 2009, 297, L1013-L1032.

27. Taraseviciene-Stewart, L.; Kasahara, Y.; Alger, L.; Hirth, P.; Mc Mahon, G.; Waltenberger, J.; Voelkel, N.F.; Tuder, R.M. Inhibition of the VEGF receptor 2 combined with chronic hypoxia causes cell death-dependent pulmonary endothelial cell proliferation and severe pulmonary hypertension. FASEB J. 2001, 15, 427-438.

28. Ciuclan, L.; Bonneau, O.; Hussey, M.; Duggan, N.; Holmes, A.M.; Good, R.; Stringer, R.; Jones, P.; Morrell, N.W.; Jarai, G.; et al. A novel murine model of severe pulmonary arterial hypertension. Am. J. Resp. Crit. Care Med. 2011, 184, 1171-1182.

29. Cretney, E.; Takeda, K.; Yagita, H.; Glaccum, M.; Peschon, J.J.; Smyth, M.J. Increased susceptibility to tumor initiation and metastasis in TNF-related apoptosis-inducing liganddeficient mice. J. Immunol. 2002, 168, 1356-1361.

30. Watt, V.; Chamberlain, J.; Steiner, T.; Francis, S.; Crossman, D. TRAIL attenuates the development of atherosclerosis in apolipoprotein E deficient mice. Atherosclerosis 2011, 215, 348-354.

31. Simmons, G.H.; Minson, C.T.; Cracowski, J.L.; Halliwill, J.R. Systemic hypoxia causes cutaneous vasodilation in healthy humans. J. Appl. Physiol. 2007, 103, 608-615.

(C) 2014 by the authors; licensee MDPI, Basel, Switzerland. This article is an open access article distributed under the terms and conditions of the Creative Commons Attribution license (http://creativecommons.org/licenses/by/3.0/). 